\title{
A Brief Discussion on the Research and Implementation of Enterprise-level Unstructured Data Management Platform
}

\author{
Zhao Li ${ }^{1, *}$, Lin Qiao ${ }^{2}$, Yanpeng Tan ${ }^{3}$, Yaozong $\mathrm{Li}^{4}$, Zhi Cao ${ }^{3}$ \\ ${ }^{1}$ ICT Department, State Grid Liaoning Electric Power Supply Co., Ltd: Shenyang 110006, China \\ ${ }^{2}$ Operation \& Maintenance Center, State Grid Liaoning Information and Communication Company: Shenyang \\ 110006 China \\ ${ }^{3}$ Technology Development Department, State Grid Liaoning Information and Communication Company: \\ Shenyang 110006, China \\ ${ }^{4}$ Financial \& Assets Department, State Grid Liaoning Electric Power Supply Co., Ltd: Shenyang 110006, \\ China \\ *Corresponding Author email: lz@ln.sgcc.com.cn
}

Keywords: Enterprise-level; unstructured data; management platform

Abstract: Operation management and business development of modern enterprises largely depend on data management, and the data includes structured data and unstructured data. Taking unstructured data as an entry point, this paper probes into the research and implementation methods of unstructured data management platform, and analyzes it from four levels, namely, business framework, application framework, integration framework and technology framework, aiming at improving the design level of enterprise- level unstructured data management platform.

\section{Introduction}

The unstructured data has many features such as format diversity, data dispersion, fast growth rate and large information, and it is an important direction for modern enterprises to realize the unified storage, analysis, management, operation and application of unstructured data through information technology and computer technology. So it is very important to study the design and implementation of unstructured data management platform.

\section{Business Framework}

The business framework of data management platform is mainly used to describe the business objectives of the management platform, which needs to be carried out according to the requirements of relevant technical regulations and management methods in the process of design. Enterprise-level unstructured data management platform includes: storage, standard, management, application and operation in accordance with business requirements.

First of all, enterprise-level unstructured data management platform needs to have unified storage function, that is, to realize the unified storage of unstructured data; this function is a basic business function of unstructured data management platform; secondly, the platform needs to have unified standard function; the platform needs to undertake the compilation of various kinds of unstructured data management and application standards within the enterprise, so as to ensure the standardization of the business work by setting up various standards (such as data platform management method, metadata writing standard, etc.); third, the unified management function of the platform: the platform management needs centralized and unified management of unstructured data, including the entire-process management of data, data asset management, etc.; fourth, the platform is equipped with unified application function; data access, processing, management and control need to be provided to the enterprise. With the premise of unified management and storage, ensure efficient and 
secure use of unstructured data; the unified operation and maintenance function of data management platform refers to the system tools that can support the routine running and maintaining of the platform, such as platform health inspection tool and unified management tool, thereby ensuring the smooth operation of the platform.

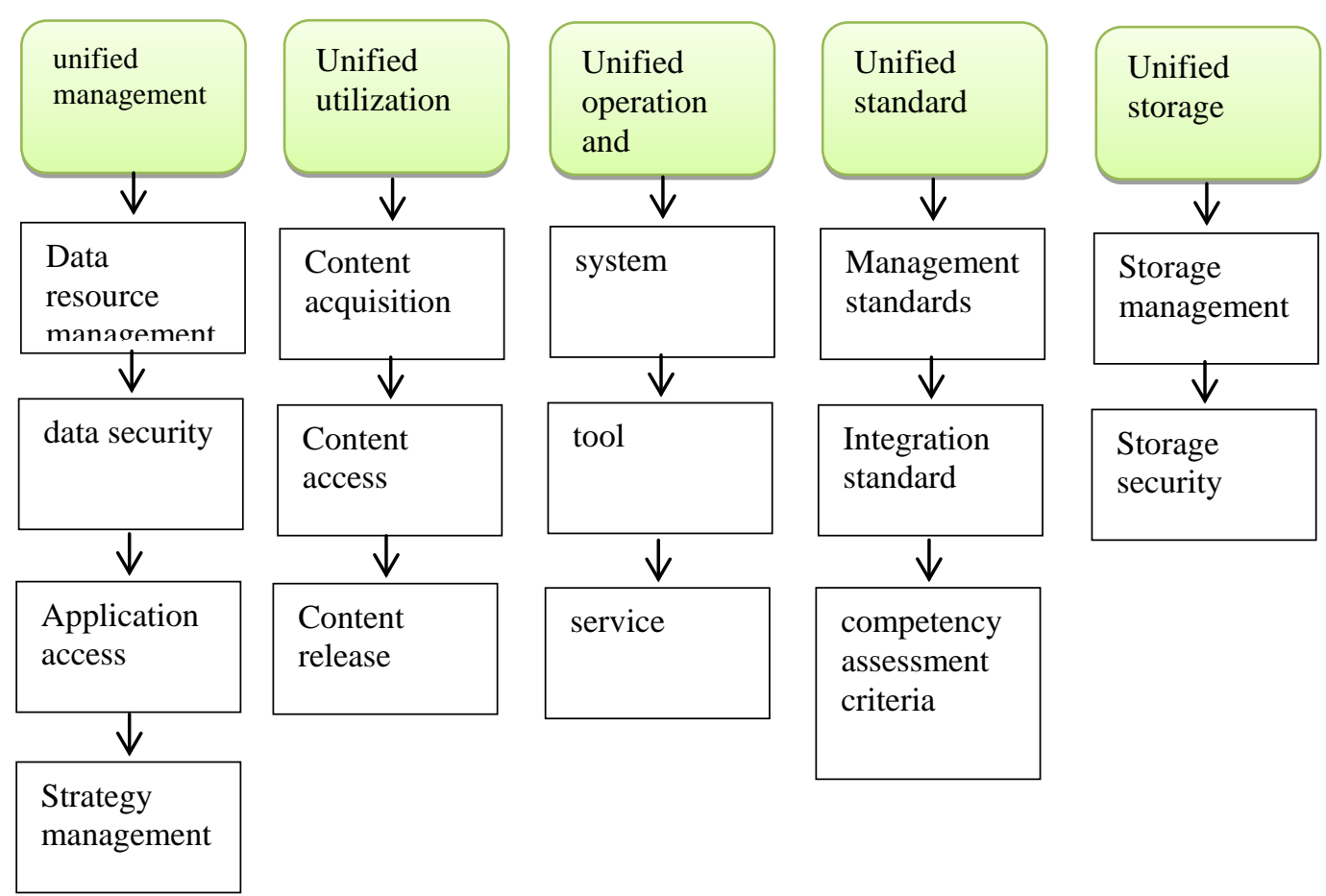

Figure 1 Business Framework of Unstructured Data Platform

\section{Application Framework}

The application framework of enterprise-level unstructured data management platform is used to describe the function of the platform and to illustrate the relationship between different functions; and the application framework is the external manifestation of the platform's technical application in business framework design.

The application framework can be divided into four parts: platform server, data management system, supporting tools and development kits. The platform management server can provide system-level services to UDS-Administrator, UDS-SDK, and UDS-Tools, including data access, transmission, processing, storage, etc. Among them, UDS-Administrator is a kind of data management system, which belongs to backstage management module in the platform, and is responsible for platform management, application, system operation, statistic report, etc. UDS-SDK Data Development Toolkit is equipped with data development function; at current stage, it can provide HTTP structure, embedded control interface and other data development toolkit; UDS-Tools is platform supporting tools, including historical data migration and other service tools.

The core of the framework design in terms of enterprise-class unstructured data management platform application is unstructured data services, which can carry out end-to-end dynamic integration of people \& data information, system application and operation process; the functional components are relatively loosely coupling, and the functional modules are independent of each other. The changes of modules will not affect other modules, so the data management platform is malleable, but also has the monitoring and alarming functions of data running. 
Table 1 Application Interface of Report

\section{Management Interface Of Report}

Data Growth List

Read Interface

io Data List

Logon Time List of the Clients

Export

Export of Logon Time List of the Clients
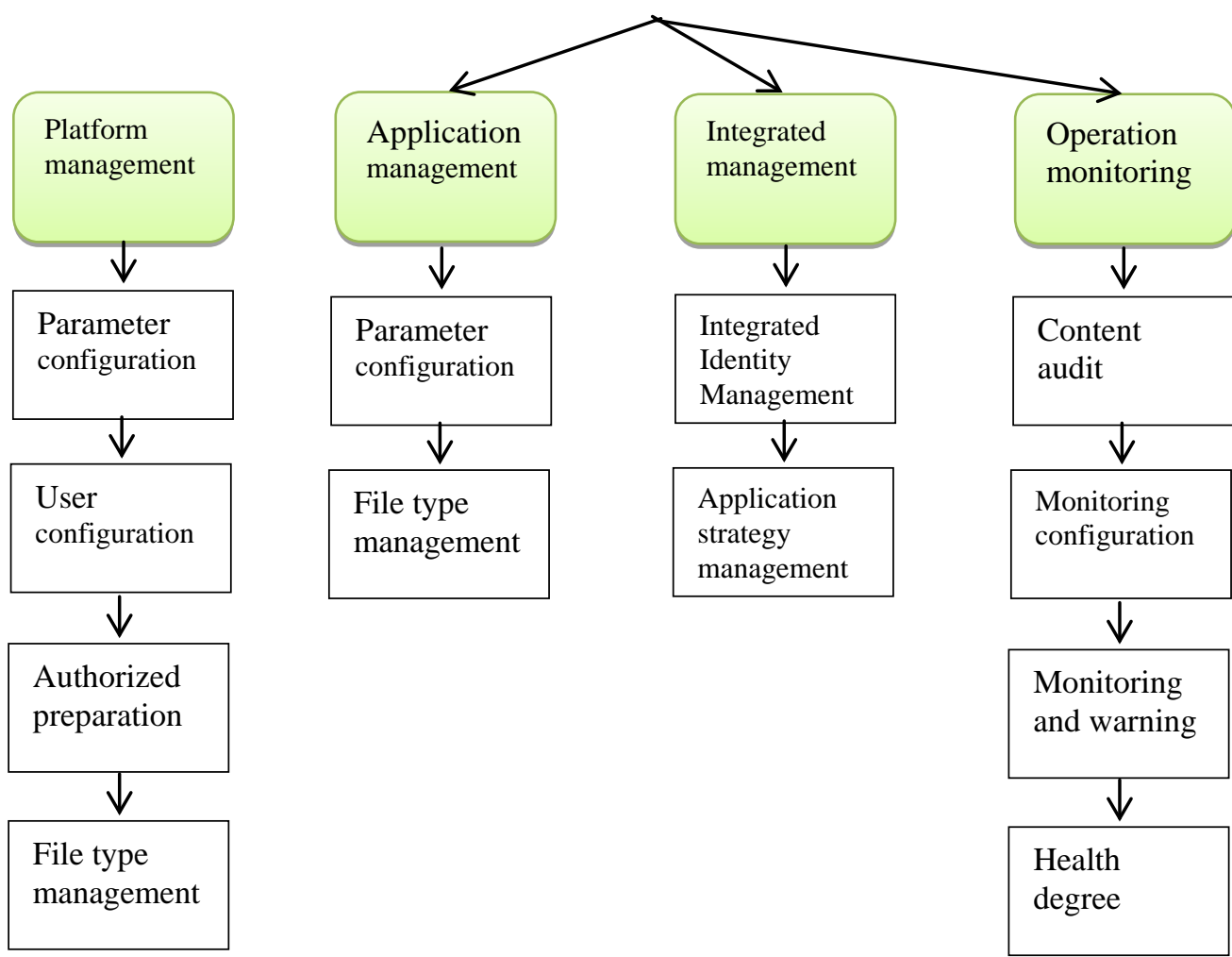

Figure 2 Application Framework of Unstructured Data Platform

Table 2 Monitoring and Alarming Functions of Data Platform

Alarm Settings Interface

Obtain SMTP Configuration Information

SMTP Settings

Set SMTP Configuration Information

Test SMTP Configuration

Obtain the List of Mailboxes And Alert Status

Sound Alarm of Turn On/Off

Alarm Settings

Mail Alarm of Turn On/Off

Add Receive Mailbox

Delete Receive Mailboxes 


\section{Integrated Framework}

Other integrated information platforms and business application systems in the enterprise operation process are all the integration goals of enterprise-level unstructured data management platform. Among them, the integration with other information platform includes unified messaging platform and unified authority platform. The integration between data management platform and unified messaging platform mainly relies on the monitoring function module of platform operation to capture its running state and system information by integrating unified messaging components and carrying out information pushes; unify authority platform, and achieve unified and integrated authentication to the management or access authority; usually, authority authentication services are delivered through client management port ${ }^{[1]}$.

Table 3 Client Management Ports

\begin{tabular}{cc}
\hline Client Management Interface \\
\hline Log In \\
Log Out \\
Client Stays Online \\
Obtain User Share List \\
Stient Interface \\
Stop Sync \\
Sync Status \\
Client File Search \\
\hline
\end{tabular}

Integration of business application systems includes unstructured data and structured data. The unstructured data can be stored and accessed in a unify manner through requesting interface service, and data information sharing and transmission can be realized; data management platform has multiplex data accesses, including HTTP, JavaAPI and other data flow integration methods, as well as page integration methods such as embedded component. Appropriate port and interface can be selected through the platform according to the business needs of an enterprise.

Table 4 Sharing Management Interface of HTTP Application Framework

\begin{tabular}{cc}
\hline Sharing Management Interface of HTTP Application Framework \\
\hline Read Interface & Obtain HTTP Sharing Configuration \\
Obtain All the HTTP Sharing Configuration \\
Create a HTTP Share \\
Write Interface \\
Create HTTP Shares in Bulk \\
Remove HTTP Shares From Directory
\end{tabular}

\section{Technical framework}

The design of the technical framework is based on the needs of function module and application technology in the data management platform, which mainly aims at describing the relationship of 
functional modules in the platform, thereby analyzing the technical components needed in the process of developing, deploying, running, and maintenance of the platform, including performance layer, business layer, access layer, data layer and interface service these five parts.

(1) Performance layer. This is the main entrance of data management platform and operation end, and also an important channel to realize human-computer interaction, which is mainly used to display platform business interface and pay attention to human-computer interaction. In the technical framework of the platform, the performance layer is positioned outermost, directly related to users, and can display relevant data, user information and other content to facilitate the user's application and operation, thereby meeting the access requirements of most browsers.

(2) Business layer. This is the core of data-management platform, but also the key joint that shows the use value of the platform, which mainly concerns about the preparation of business rules and the realization of business process; the business layer is directly related to the logical field of the data-management platform compared with other layers. In the data-management platform, business layer belongs to a weak-coupling structure, which plays a transition role between the access layer and the performance layer.

(3) Access layer. It can provide data access function, and facilitate the operator to visit the database and the document resources, while simultaneously carrying on data inquiry, retrieval, input, deletes and other operations.

(4) Data layer. It mainly includes relational data and document database, which is the storage part of data information.

(5) Interface service. With the application of Web service technology, it is independent of the interface of management platform and programming language software, which can realize data exchange through XML ${ }^{[2]}$.

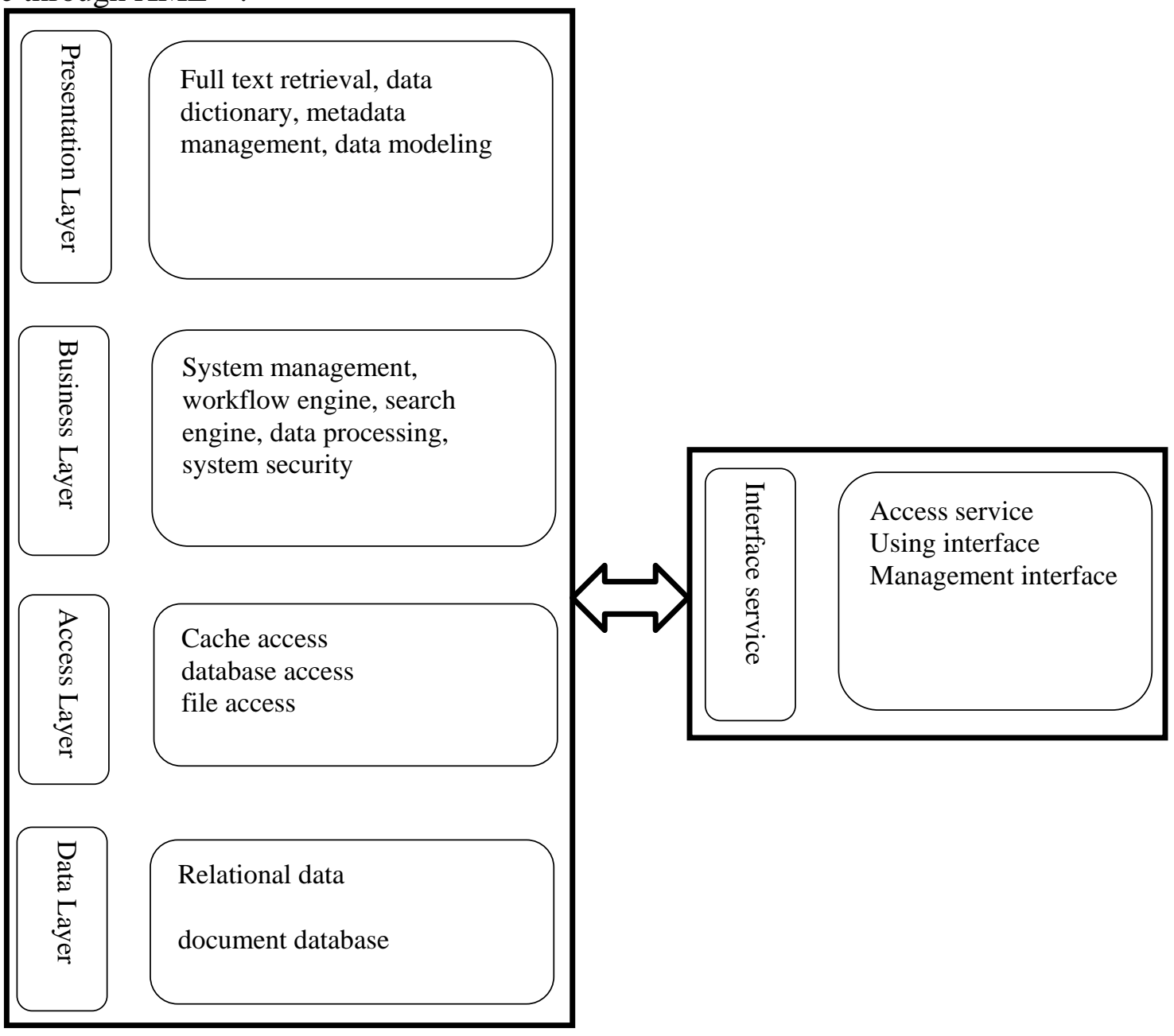

Figure 3 Technology Framework of Unstructured Data Management Platform 


\section{Conclusion}

With the rapid development of information technology, enterprises are more and more aware of the importance of effective management of unstructured data. In the process of enterprise operation and development, the construction of data management platform is carried out with more deliberately. In the process, the specific requirements of the relevant technical application norms, laws and regulations need to be strictly followed.

\section{References}

[1] Rasmus S M. Indigenizing CBPR: Evaluation of a Community-Based and Participatory Research Process Implementation of the Elluam Tungiinun (Towards Wellness) Program in Alaska [J]. American Journal of Community Psychology, 2016, 54(1-2):170-179.

[2] Raiten D J, Neufeld L M, Deregil L M, et al. Integration to Implementation and the Micronutrient Forum: A Coordinated Approach for Global Nutrition. Case Study Application: Safety and Effectiveness of Iron Interventions.[J]. Advances in Nutrition, 2016, 7(1):135-148. 